\title{
When was the last time you induced general anesthesia for cesarean section?
}

\author{
Hiroyuki Sumikura
}

Received: 30 January 2015 / Accepted: 3 February 2015 / Published online: 18 February 2015

(C) Japanese Society of Anesthesiologists 2015

There is a common saying that truth never grows old. In the field of obstetric anesthesia, however, several truths have become old over the past decade [1]. For example, ephedrine is no longer the first-line treatment for hypotension after spinal anesthesia for cesarean section, and spinal anesthesia is no longer contraindicated in patients with pre-eclampsia. On the other hand, the dogma that 'general anesthesia for cesarean section is riskier than neuraxial anesthesia and should be avoided' has been a firm belief to date, but the survival of this dogma for another decade is questionable.

1. Current status of general anesthesia for cesarean sections. Hawkins et al. [2] reported in 1997 that the case fatality ratio for general anesthesia for cesarean section was 16.7 [95\% confidence interval (CI), 12.9-21.8] times that for regional anesthesia between 1985 and 1990. Since that report, every effort has been made to avoid general anesthesia for cesarean section, with the rate of use falling to the lowest in the United States. For example, this rate is reported to be $<1 \%$ at Brigham and Women's Hospital in Boston, which is ironic because one of the most prominent educational hospitals for obstetric anesthesia cannot provide their residents with the opportunity to perform cesarean section under general anesthesia [3]. Although $1 \%$ is rather extreme, extensive data from several educational hospitals have revealed a value of as low as $5 \%$ in the United States [4]. On the other hand, some countries

H. Sumikura $(\triangle)$

Department of Anesthesiology and Pain Management,

Juntendo University School of Medicine, 3-1-3 Hongo,

Bunkyo-ku, Tokyo, Japan

e-mail: hiroyuki.sumikura@gmail.com still prefer general anesthesia as a standard technique for cesarean section. In addition, several studies reporting the use of general anesthesia for cesarean section are submitted to the Journal of Anesthesia from these countries $[5,6]$. The increased rate of use in developing countries may be explained by their strict indications for cesarean sections. In addition, their limited medical resources and insufficient medical education may play a role.

In contrast to the status in these developed and developing countries, the current status of general anesthesia for cesarean section in Japan remains unclear [7]. First, it should be noted that obstetricians manage anesthesia in $>50 \%$ cases and it is natural that they opt for spinal anesthesia. On the other hand, some anesthesiologists who participate in cesarean sections prefer general anesthesia. This may be explained by several reasons, such as a lower medical fee for spinal anesthesia than for general anesthesia, the lack of popularity of epidural analgesia during labor, and the underdevelopment of subspecialty education in the field of obstetric anesthesia. As a result, the rate of general anesthesia use for cesarean section remains relatively high in Japan compared with that in other developed countries. However, it is interesting to note that no case of anesthesia-related maternal death has been identified in a national survey of the causes of maternal death since 2010; this implies the need to revaluate the validity of the above-mentioned dogma.

2. Should efforts to avoid general anesthesia for cesarean sections continue? In 2011, Hawkins et al. [8] updated their previous study and reported that the case fatality rate for general anesthesia for cesarean section decreased dramatically from 16.8 per million in 19911996 to 6.5 per million in 1997-2002, whereas that for 
regional anesthesia slightly increased from 2.5 to 3.8 per million, respectively. This resulted in a decrease in the case fatality ratio for general anesthesia to 1.7 times that for regional anesthesia, with no significant difference between the two techniques (95\% CI, 0.6-4.6). However, a selection bias should be considered here, because there was a large shift from general to regional anesthesia for high-risk patients. In addition, the overall anesthesia-related maternal mortality rate decreased to nearly $60 \%$ when data from 1979-1990 were compared with those from 1991-2002. These findings indicate the increased safety of anesthesia for cesarean section, including general anesthesia, in the more recent decades. This improvement should be attributed to the development of airway devices such as supraglottic airways, monitoring devices such as oxygen saturation monitors and capnometers, and protocols for difficult airway management.

3. Management of general anesthesia for cesarean section. The decreased use of general anesthesia for cesarean section in developed countries reduced the number of opportunities for residents to practice this technique [9]. Furthermore, the optimal management of general anesthesia using up-to-date technology, drugs, and knowledge was not well studied during its period of unpopularity. Therefore, it was considered valuable to reconfirm the current consensus regarding general anesthesia for cesarean sections.

From these perspectives, the editorial committee of the Journal of Anesthesia hosted a symposium titled "Current Consensus of General Anesthesia for Cesarean Section" in the Annual Meeting of the Japanese Society of Anesthesiologists in 2014. In the first half of the symposium, the validity of the dogma was discussed after two keynote lectures. Dr. Sato from the National Center for Child Health and Development, where the rate of general anesthesia use is $<5 \%$, presented his efforts to avoid general anesthesia for cesarean sections, while Dr. Niwa from Hirosaki University, where the rate is $>50 \%$, presented an audit of his practice. In the latter half, the optimal management of general anesthesia during cesarean section was discussed after three lectures on airway management in parturients by Dr. Asai from Dokkyo University, on maintenance of general anesthesia during cesarean section by Dr. Hagihira from Osaka University, and on pain relief after cesarean section by Dr. Nakamoto from Osaka Rosai Hospital. The symposium was successful, with a capacity audience and a heated dispute.
4. Policy of the Journal of Anesthesia regarding general anesthesia for cesarean section. The current development of technology and knowledge requires evaluation of the validity of the previously mentioned dogma and discussion of the optimal management of general anesthesia for cesarean section. As a section editor for papers on obstetric anesthesia published in the Journal of Anesthesia, I can assure you that we will provide a platform for these evaluations and discussions. As a first step, the contents of the JA symposium in 2014 will be published as a review article. In addition, we will accept manuscripts reporting the use of general anesthesia for cesarean sections if their study protocols are approved by an appropriate ethics committee and they are worthy of publishing. Furthermore, suitable reports of cases managed by general anesthesia will be accepted for publication either in the Journal of Anesthesia or in the JA Clinical Reports, which will be launched this year. We hope these steps will strengthen our policy of providing a scientific discussion that is free of dogmatism.

\section{References}

1. Staikou C, Paraskeva A, Karmaniolou I, Mani A, Chondrogiannis K. Current practice in obstetric anesthesia: a 2012 European survey. Minerva Anestesiol. 2014;80:347-54.

2. Hawkins JL, Koonin LM, Palmer SK, Gibbs CP. Anesthesiarelated deaths during obstetric delivery in the United States, 1979-1990. Anesthesiology. 1997;86:277-84.

3. Palanisamy A, Mitani AA, Tsen LC. General anesthesia for cesarean delivery at a tertiary care hospital from 2000 to 2005: a retrospective analysis and 10-year update. Int J Obstet Anesth. 2011;20:10-6.

4. D’Angelo R, Smiley RM, Riley ET, Segal S. Serious complications related to obstetric anesthesia: the serious complication repository project of the Society for Obstetric Anesthesia and Perinatology. Anesthesiology. 2014;120:1505-12.

5. Demiraran Y, Albayrak M, Yorulmaz IS, Ozdemir I. Tramadol and levobupivacaine wound infiltration at cesarean delivery for postoperative analgesia. J Anesth. 2013;27:175-9.

6. Eslamian L, Jalili Z, Jamal A, Marsoosi V, Movafegh A. Transversus abdominis plane block reduces postoperative pain intensity and analgesic consumption in elective cesarean delivery under general anesthesia. J Anesth. 2012;26:334-8.

7. Yamada Y. Obstetric anesthesia: from historical perspective to current practice—preface and comments. Masui. 2010;59:290-2.

8. Hawkins JL, Chang J, Palmer SK, Gibbs CP, Callaghan WM. Anesthesia-related maternal mortality in the United States: 19792002. Obstet Gynecol. 2011;117:69-74.

9. Searle RD, Lyons G. Vanishing experience in training for obstetric general anaesthesia: an observational study. Int $\mathrm{J}$ Obstet Anesth. 2008;17:233-7. 